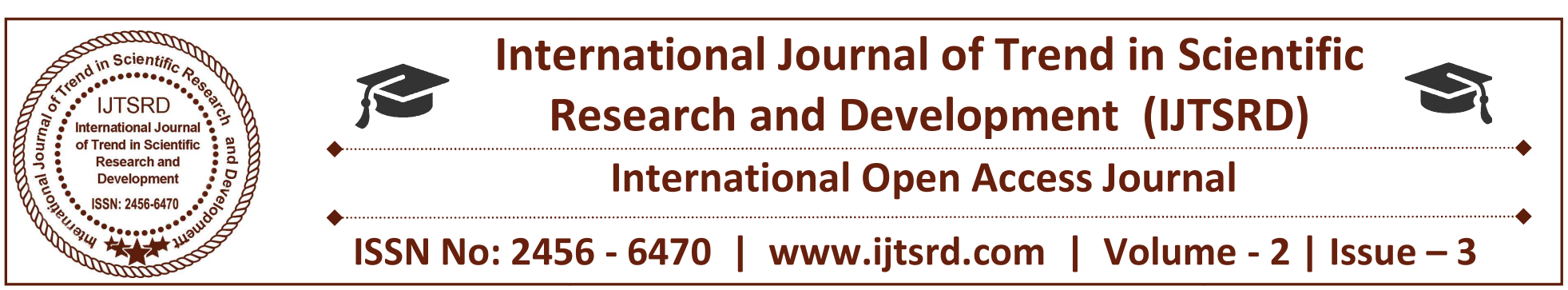

\title{
Facial Authentication for Controlling Mouse Cursor with Hand Motion Detection using Webcam
}

\author{
T. Manigandan ${ }^{1}$, S. Rohit ${ }^{2}$, R. Prakash ${ }^{3}$ \\ Department of Computer Science and Engineering, \\ G.K.M. College of Engineering and Technology, Chennai, Tamil Nadu, India
}

\begin{abstract}
In this paper, a human computer interface using hand gestures or any hand signal is introduced. This system is enhanced to replace the use of computer mouse with any hand signal instead of using any face expression. The system track the users hand movements with a webcam to displacement of the mouse cursor and users any hand sign is used to translates them into mouse click events on computer screen. Along with this system we also introduce facial authentication for privacy before some movements are introduced. Finally the system thus eliminates the direct contact with the mouse. Hand Motion Detection is used in various aspects and the results have been tremendously gathered. So HCI profound to be used in virtual basis and important techniques can be gathered.
\end{abstract}

Keywords: Human Computer Interaction, Image processing, Facial Recognition, Hand Gesture Recognition

\section{INTRODUCTION}

Human Computer interaction(HCI) is the basis that interaction is between the user and the computer. This is when the user design and implementation held in some cases. It also has a task which performs on the basis of user analysis and also defines the interface. Moreover Image Processing requires the input from the user. It works according when the user enhanced to provide images without any possible chances for other displacing substance in that image before processing is done. For more Purpose Facial Recognition can be introduced for accessing only private section or other important work related source.

Facial Recognition are introduced in various sources and different techniques which corresponds to access them in a vast area. Hand motion detection is used when the user performs some movement with their hands like hand signals are found to be in various fields and any information from it can be easily obtained. Facial and Hand Gesture Recognition can be obtained when the user source is very effective and found to be used only in a privacy section.

\section{RELATED WORKS}

The field of Facial Recognition are more generally used in smart devices for privacy part which can be an application or some resources. For such cases facial Recognition used in the application like social network facebook which can be maintained as private section which were introduced. Moreover Dynamic Facial Emotion Recognition Oriented to HCI applications was proposed by Samuel Marcos Pablos[1] can be the basis of HCI. Hand Gesture Recognition were introduced in various parts of the System based on computer and the users interaction. Some of them are used in Wavelet Invarient Moments which was proposed by Xi Liu[2] and Hand Gesture Recognition System were used with Software application such as Opencv and visual Studio $\mathrm{C}++[3]$.Another work in Hand Gesture Recognition with Leap Motion Controller which they were using the HCNF -based classifier[4]. Though the use of these Hand Gesture Recognition system which were introduced is to maintain a normal source of computer and human interaction purpose environment only. Further related work can be Interactive Projector Screen with hand detection using gestures in which 
movement of the cursor was proposed and for more convenient it has to be used with the help of tracking gloves and the fingertip detection[5].Both Facial as well as Hand Gesture Recognition uses many various classifiers which can be useful in future purpose and to develop an unique environment from these technique. It is to be sure that only convenient related works are only used in the field of computer society and thus in HCI basis a vast environment can be acquired.

\section{PROPOSED SYSTEM}

Many works have been done on hand gesture recognition with different techniques. The proposed system of hand gesture recognition uses hand sign's instead of using any input devices to control the mouse cursor. Along with the hand gestures, we also introduce user authentication by facial recognition. The Facial Recognition is only to be accessed by the user authentication purpose only because other images are invalid.

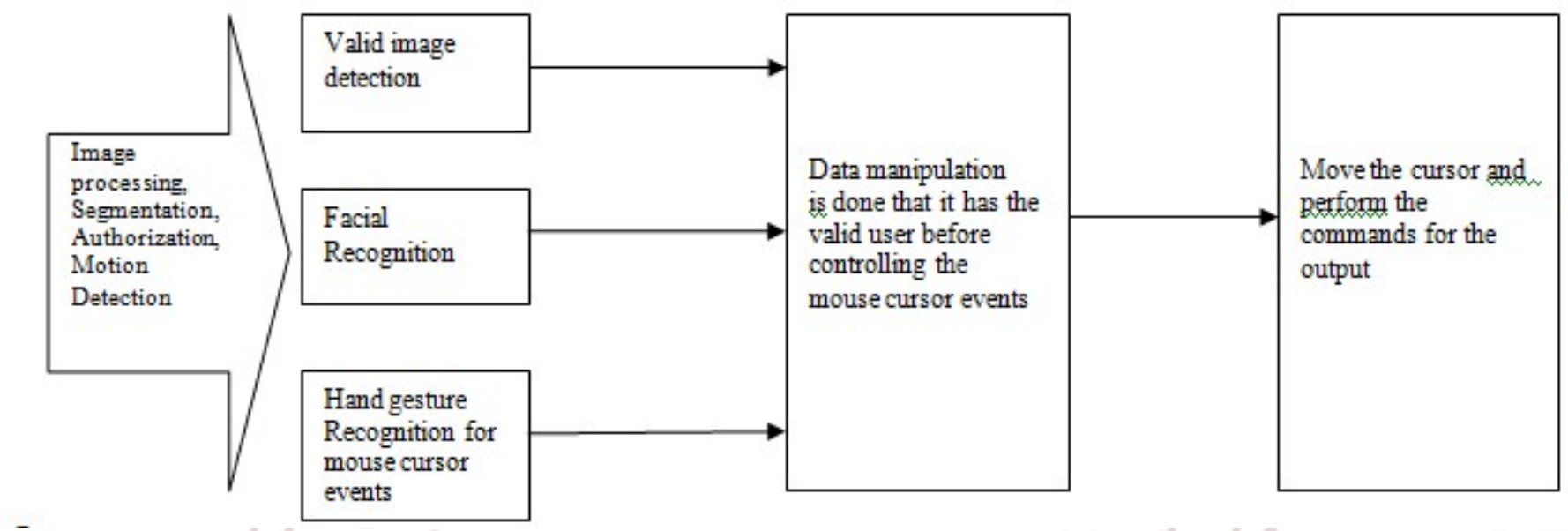

\section{A. Pre processing}

has been segmented and thus it analyse the image and act further accordingly to the users respond by which

Pre processing is an operation with images both as an input level and output level. The image is cropped to remove outer parts of an image for improve framing, and then it is checked for sudden unwanted distortions. For more aspect pre processing also validates only users given image. The major advantage is that the image can be retrieved for further number of times when the user doesn't change the image from the data.

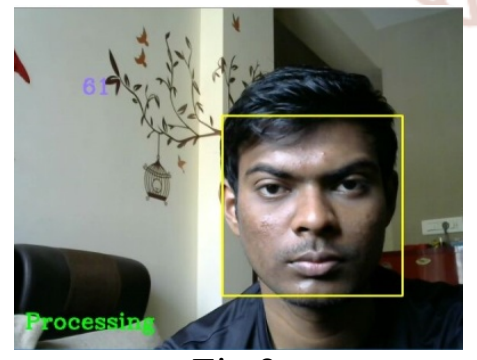

Fig.2

\section{B. Segmentation}

Segmentation is in order to detect objects or divide the images into regions. The second step is Image segmentation which is for image analysis. The result of image segmentation is a set of segments which collectively covers the entire image. when the image the user has given only the valid image for segmentation process.

Moreover Segmentation can be done in various techniques which uses only the exact region of the particular image that has to be Segmented.

\section{Authorization}

Authorization is the function of security mechanism to access levels of user related system resources. Only the particular User/client can have the right to access their system resource. In this proposed manner facial authentication or authorization is implemented for recognises the facial of the user and thus performs some task that the user has to do. It is generally used for the privacy and security purpose by which the user has the access.

Authorization is the process of users identity before accessing or retrieving data the from the system or storage memory. For this purpose facial Recognition is been introduced for accessing the data or other 
activities that the user wants to perform. In general authentication is used in various sources like social network etc., Thus the user can able to access or even perform some actions for some purpose.

\section{Motion Detection}

Motion detection is to detect the captured image as input with the help of several methods such as optics which is used for video and camera systems. It is action of sensing a physical movement in given area. In this process the users hand motion is to be detected and then performs some task such as controlling of the mouse cursor. The user moves the cursor from one place to the other and even implements some hand gestures for mouse click events. Very few possible chances that it is inconvenient to use.

\section{Disadvantages}

People with hand problems cannot access this kind of system. Provide access only to the users. Webcam is mandatory

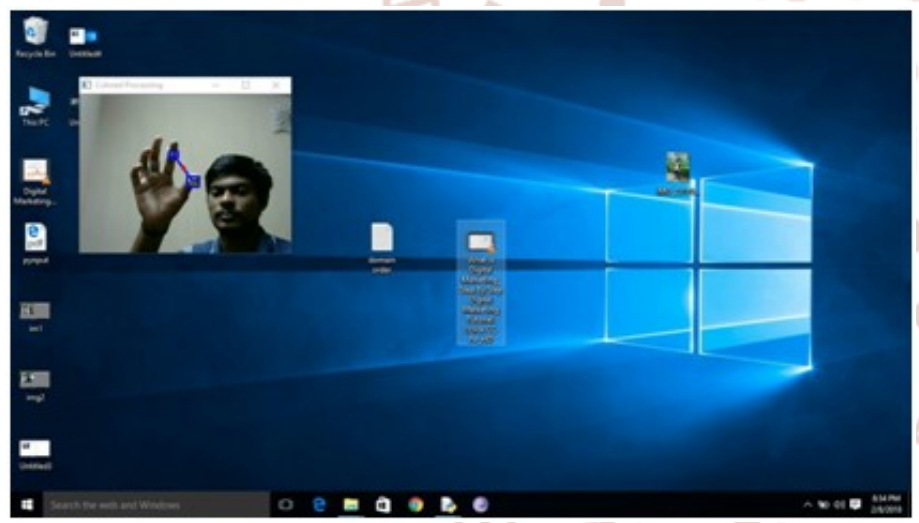

Fig. 3

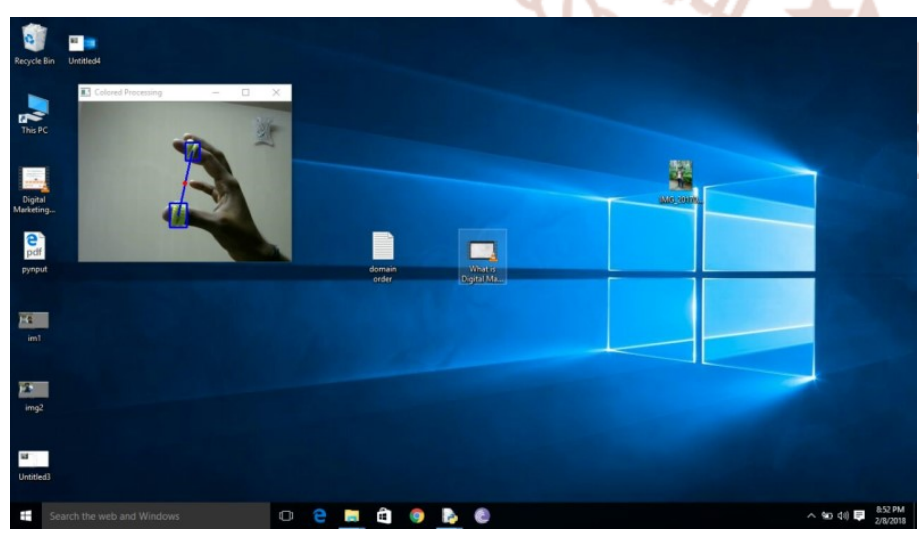

Fig.4

\section{CONCLUSIONS}

Thus the proposed system further detailed about the facial Recognition for Authentication purposes and also hand Gesture Recognition for controlling the mouse cursor by giving any gestures for click events. While using this system the user should the better convenient webcam before implementing this process by means of high resolution webcam is very important. It is very useful presenting in MS powerpoint and also in gaming. In $\mathrm{HCI}$, various implementations is provided to enhance rather than using the hardware devices. Thus Facial Recognition and Hand Gesture Recognition in computer science field have a vast area for implementing new techniques in the future.

\section{REFERENCES}

1. Samuel Marcos Pablos, Jaime Gómez GarcíaBermejo, Eduardo Zalama Casanova and Joaquín López "Dynamic Facial Emotion Recognition Oriented to HCI Applications", Published by Oxford University Press on behalf of The British Computer Society.

2. Xi Liu, Chen Li, Lihua Tian, "Hand Gesture Recognition based on Wavelet Invariant Moments",IEEE International Symposium on Multimedia 2017.

3. Prof. Praveen D. Hasalkar, Rohit S. Chougule, Vrushabh B. Madake, Vishal S. Magdum," Hand gesture recognition system",published in IJARCCE, 2015.

4. Wei Lu, Member, IEEE, Zheng Tong, and Jinghui Chu," Dynamic Hand Gesture Recognition With Leap Motion Controller",

5. IEEE SIGNAL PROCESSING LETTERS, VOL. 23, NO. 9, SEPTEMBER 2016.

6. Rishabh Sharma, Raj Shikher, Nutan V. Bansode and Prachi R. Rajarapollu," Interactive Projector Screen with Hand Detection Using Gestures", 2016 International Conference on Automatic Control and Dynamic Optimization Techniques (ICACDOT)

7. K S Chidanand Kumar, "A Vision based Application for Virtual Mouse Interface Using Finger-Tip," IJCSIT, vol. 3 (3), 2009

8. Dhawan, V. Honrao, "Implementation of Hand Detection based Techniques for Human Computer Interaction," International Journal of Computer Applications, vol. 72, no. 17, 2013.

9. A. Argyros and M. I. A. Lourakis, "Vision-based interpretation of hand gestures for remote control 
of a computer mouse," in Proc. ECCV, 2006, pp. $40-51$.

10. S. Kang, C. Kim, and W. Son, ' Developing userfriendly hand mouse interface via gesture recognition," in Proc. Conf. Korean Soc. Broadcast Eng., 2009, pp. 129-132.

11. V. H. Amiraj Dhawan, "Implementation of hand detection based techniques for human computer interaction," International Journal of Computer Applications, vol. 72, 2013.

12. T. S. Attila Licsr, "Hand gesture recognition in camera-projector system" ,Uni- versity of Veszprm, Department of Image Processing and Neurocomputing, 2004

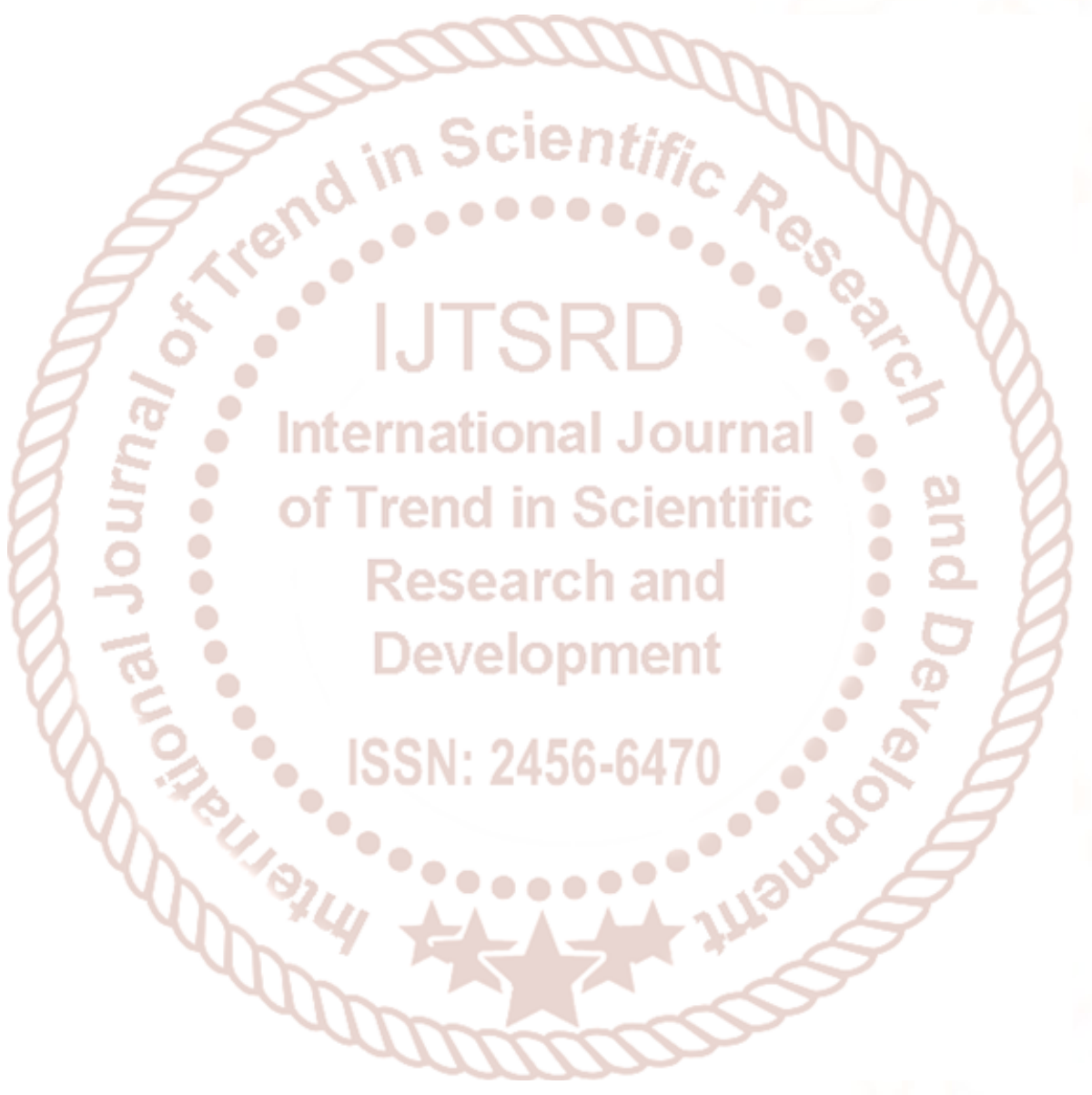

